Results 8 papers and reports which contributed to the final model. The proportional meta-analysis showed a pooled proportion positive for chlamydia of $7.7 \%$ (95\% CI $5.2 \%$ to $10.6 \%$ ). All the studies were reported on $<24$ years age group and there was only limited data on males. Hence no separate analyses were performed according to age group or gender. Chlamydia screening programs in community pharmacies tend to be targeted at certain client groups for example, young people, those seeking emergency contraception in pharmacies. Studies reviewed reported that clients and pharmacists find chlamydia services via community pharmacy broadly acceptable. However the uptake of the service was much lower than expected and tended not to include men and ethnic minorities.

Conclusion The reported prevalence of chlamydia infection in pharmacy setting is similar to estimates from general practice thus giving wider choice of care to young people. This new approach is acceptable to both young people and pharmacists. Encouraging men and ethnic minorities to access community pharmacy based chlamydia services remains a challenge.

\section{SP6-46 RISK PERCEPTION OF SMOKING AND OUITTING IN HUNGARY}

doi:10.1136/jech.2011.142976q.17

${ }^{1} \mathrm{E}$ Paulik, ${ }^{*}{ }^{1} \mathrm{M} N$ Ágnes, ${ }^{1} \mathrm{~N}$ László, ${ }^{2} \mathrm{D}$ Easterling, ${ }^{3} \mathrm{~T}$ Rogers. ${ }^{1}$ Department of Public Health, Faculty of Medicine, University of Szeged, Szeged, Hungary; ${ }^{2}$ Wake Forest University School of Medicine, Winston-Salem, USA; ${ }^{3}$ Public Health Institute, Oakland, USA

Introduction Despite improvements in tobacco control smoking is the leading, preventable risk factor for premature death and disability in Hungary. The purpose of this study was to identify the determinants of smoking and quitting in Hungary.

Methods The first wave of a quantitative longitudinal study was delivered in 2009. A sample of individuals $(n=2250)$ aged $16-70$ years was selected from the seven geographical regions of Hungary. The survey was conducted through self-administered questionnaires. $\chi^{2}$ test and one-way ANOVA were applied to compare smokers who attempt to quit with smokers without intentions.

Results One-third (33.2\%) of interviewed were current smokers, $17.6 \%$ were ex-smokers, and $49.1 \%$ non-smokers; the prevalence of smoking was significantly higher in men and lower educated respondents. Actually $48.0 \%$ of smokers wanted to quit smoking, $29.5 \%$ didn't and $22.5 \%$ was uncertain. Age, gender, education and marital status had no effect on behaviour, but the knowledge about the various risks of smoking $(p<0.001)$ and the support for tobacco control in public places $(p<0.001)$ were significantly higher among smokers who wanted to quit smoking.

Conclusion The improvement of knowledge and the implementation of anti-smoking policy can support the decision making about quitting smoking independently from socio-demographic factors.

This publication was made possible by Grant Number 1 R01 TW007927-01 from the Fogarty International Center, the National Cancer Institute, and the National Institutes on Drug Abuse, within the National Institutes of Health ( $\mathrm{NIH})$. Its contents are solely the responsibility of the authors and do not necessarily represent the official view of the $\mathrm{NIH}$.

\section{SP6-47 MISCONCEPTIONS ABOUT HUMAN PAPILLOMAVIRUS IN A SAMPLE OF HUNGARIAN ADOLESCENT GIRLS}

doi:10.1136/jech.2011.142976q.18

${ }^{1}$ A Varga-Tóth, * ${ }^{2}$ E Paulik. ${ }^{1}$ Obstetrics and Gynecology Department, Faculty of Medicine, University of Szeged, Szeged, Hungary; ${ }^{2}$ Department of Public Health, Faculty of Medicine, University of Szeged, Szeged, Hungary

Introduction The high mortality of cervical cancer is a public health problem in Hungary. The risk of cervical cancer is increased by the human papillomavirus (HPV) infection. The awareness of HPV associated diseases and HPV vaccination plays an important role in the prevention. The purpose of this survey was to assess the knowledge about HPV and its prevention among adolescent girls.

Methods A self-administered questionnaire concerning knowledge of HPV, cervical cancer and vaccines were applied. Altogether 589 girls (aged 14-18) taking part in a short course about sexual and reproductive health were involved; the questionnaires were completed before the education. SPSS for Windows version 17.0 was used to analyse the results.

Results Half of the girls were sexually active, $75.6 \%$ of them began sexual activity at $15-17$ ages; the mean age was 15.39 years. Only $49.9 \%$ of the respondents identified correctly that HPV is a sexually transmitted infection. The girls being familiar with this fact were more informed about the various ways of HPV transmission. In general, the awareness of HPV transmission was very low $(0.8 \%$ gave totally correct answer). $34.8 \%$ of the sexually active girls thought that using a condom completely prevent HPV infection. $47.5 \%$ have heard about the HPV vaccine.

Conclusion This survey indicated that adolescent girls have low level knowledge about HPV, although they are sexually active. Serious misconceptions exist about specific aspects of the disease. Improvements in the sexual education in the upper primary schools are needed to encourage the prevention of cervical cancer in Hungary.

\section{SP6-48 NUTRITIONAL REQUIREMENTS FOR CHILDREN AGED 2-5 YEARS}

doi:10.1136/jech.2011.142976q.19

G França, ${ }^{*}$ D Gigante, C Dutra. Universidade Federal de Pelotas, Pelotas, Rio Grande do Sul, Brazil

Objective To produce recommendations for children aged 2-5 years, considering the specificities of this age group in relation to nutritional needs and capacity of food intake.

Methods Individual energy requirements were estimated using the formulae for calculating the Recommended Dietary Intake. Taken as reference data on weight and height, by age and sex, referring to the 50th percentile established by the multicenter study of growth references developed by the WHO (2006). After obtaining the total energy, we calculated its mean and SD for each year studied. The minimum and maximum portions related to the lower and higher energy values established were adapted in the Food Guide Pyramid developed by Philippi et al, 1996.

Results The average total energy was around 1000, 1300 and $1400 \mathrm{kcal}$ for two, three and 4 years old children, respectively. Minimum and maximum portions were: 4 to 7 servings for the group of cereals, breads, tubers and roots, 3 to 4 for vegetables, 4 to 5 for fruit and 3 servings for milk and milk products, 2 for meat and eggs; 1 part for the group of pulses, 1 to 2 for oils and fats, and 1 to 2 servings for sugars and sweets.

Conclusion The results of this study can be applied in nutritionist's professional practice and to planning public policies aimed at ensuring the adequate provision of nutritional support to children in the age group studied.

\section{SP6-49 EVALUATION OF THE DEFICIENCY OF VITAMIN D IN A POPULATION OF WOMEN IN BRAZIL: PILOT STUDY}

doi:10.1136/jech.2011.142976q.20

J H Yang, R V Silva, C A Borges, C de Aguiar Martins, M C R de Stefano, M A de Faria Pádua. * Clinica Synesis, São Paulo, São Paulo, Brazil

Introduction Vitamin D serum dosage evaluation in 40 women submitted to the WHO Healthy Life Quality-SHORT FORM 36 (SF-36). 